DOI: $10.24850 /$ j-tyca-2021-02-12

Notas

\title{
Caracterización morfométrica de dos cuencas altoandinas del Perú utilizando sistemas de información geográfica
}

\section{Morphometric characterization of two high Andean basins of Peru using geographic information systems}

Sandra del Águila1, ORCID: https://orcid.org/0000-0001-8051-3575 Abel Mejía², ORCID: https://orcid.org/0000-0002-9070-3898

${ }^{1}$ Universidad Nacional San Cristóbal de Huamanga, Ayacucho, Perú; Universidad Nacional Agraria La Molina (UNALM), Programa de Doctorado en Recursos Hídricos, Lima, Perú, sandra.delaguila@unsch.edu.pe 2Universidad Nacional Agraria La Molina (UNALM), Programa de Doctorado en Recursos Hídricos, Lima, Perú, jabel@lamolina.edu.pe

Autora para correspondencia: Sandra del Aguila, sandra.delaguila@unsch.edu.pe

\section{Resumen}

La caracterización morfométrica de cuencas hidrográficas es importante, porque permite describir procesos geomorfológicos e inferir 


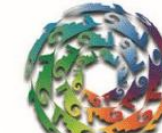

Ciencias $\overline{\text { Tagua }}$

comportamientos hidrológicos: caudales máximos, erosión del suelo y recarga de agua subterránea. En los países en desarrollo, los estudios sobre morfometría de cuencas son escasos; no obstante, su aplicación es sustancial, sobre todo en regiones no instrumentadas. Se realizó un análisis morfométrico evaluando las características de drenaje de dos cuencas de ríos altoandinos, en la región Junín, Perú: la cuenca del río Anya, en el lado oriental de la cordillera, y la del río Mchique, en el flanco occidental de los Andes centrales. Se delimitaron las cuencas y se calcularon los parámetros morfométricos de relieve, forma y drenaje, utilizando sistemas de información geográfica (SIG). Ambas cuencas son de orden 3 con patrones de drenaje diferenciados, siendo la cuenca Anya dendrítica y Mchique subdendrítica, con influencia de elementos estructurales, dada su forma alargada particular. Los valores bajos de densidad de drenaje $\left(D_{d}\right)$ fijan a las cuencas como de altas tasas de infiltración, sin embargo, éstos responden a sus ubicaciones en cabeceras. Los valores de elongación $(E)$ implican una forma alargada para Anya y más para Mchique, lo cual indica una menor vulnerabilidad a inundaciones repentinas $y$, consecuentemente, una gestión de avenidas más sencilla. El alto factor topográfico $\left(F_{t}\right)$ de las cuencas es indicador de pendientes pronunciadas $y$, por consiguiente, de mayor intensidad de procesos erosivos. Los resultados pueden ser base de estudios hidrológicos e hidráulicos, manejo de cuencas y gestión de recursos hídricos en la zona.

Palabras clave: cuencas hidrográficas, hidrología, SIG, MED.

\section{Abstract}


The morphometric characterization of watersheds is important because it allows to describe geomorphological processes and infer hydrological behaviors: maximum flows, soil erosion and groundwater recharge. In developing countries, studies on watershed morphometry are scarce; nevertheless, its application is substantial, especially in non-instrumented regions. A morphometric analysis was carried out evaluating the drainage characteristics of two high Andean river basins in the Junin region, Peru: the Anya river basin on the eastern side of the mountain range and the Mchique River on the western flank of the central Andes. The basins were delimited and the morphometric parameters of relief, shape and drainage were calculated, using Geographic Information Systems (GIS). Both basins are of order 3 with differentiated drainage patterns, with the Anya dendritic basin and Mchique subdendritic with influence of structural elements given their particular elongated shape. The low values of drainage density $\left(D_{d}\right)$ fix the basins as having high infiltration rates, however, they respond to their headland locations. The values of elongation $(E)$ imply an elongated shape for Anya and more for Mchique, which indicates a lower vulnerability to flash floods and, consequently, a simpler flood management. The high topographical factor $\left(F_{t}\right)$ of the basins is an indicator of steep slopes and, consequently, of greater intensity of erosive processes. The results can be the basis for hydrological and hydraulic studies, watershed management and water resources management in the area.

Keywords: Watersheds, hydrology, GIS, DEM.

Recibido: 14/06/2019 
Teccnología y

Ciencias $₫$ Agua

Aceptado: 06/07/2020

\section{Introducción}

En regiones áridas y semiáridas, la escorrentía de las montañas puede ser la fuente principal del agua en las partes bajas (Liniger, Weingartner, \& Grosjean, 1998), siendo las características morfológicas de las cuencas hidrográficas determinantes en sus comportamientos hidrológicos (Cruz, Gaspari, Rodriguez, Carrillo, \& Telles, 2015; Vieceli et al., 2015).

La morfometría es la medida y el análisis matemático de la configuración de la superficie terrestre (forma, dimensiones de accidentes geográficos, red de canales y pendiente del terreno); por lo que es un principio ampliamente reconocido que la morfología de la cuenca de drenaje refleja aspectos geológicos y procesos geomorfológicos a lo largo del tiempo (Horton, 1945; Strahler, 1964; Miller, 1953; Jardí, 1985;).

Hoy en día, la caracterización morfométrica de las cuencas hidrográficas se realiza con un sistema de información geográfica (SIG), ya sea de modo manual o automático; es ideal por ser dinámico en la visualización y procesamiento de la cuantificación de los atributos topográficos de una cuenca (Medeiros et al., 2019; Kabite \& Gessesse, 2018; Rai, Mohan, Mishra, Ahmad, \& Mishra, 2017). Los estudios han demostrado que los datos sobre parámetros morfométricos y descargas 
de ríos de las cuencas de drenaje en los países en desarrollo escasean o son muy inadecuados cuando existen (Oruonye, Ezekiel, Atiku, Baba\& Musa, 2015). En los ríos del Perú, son pocos los estudios desde la perspectiva hidrogeomorfológica (García \& Otto, 2015).

Las cuencas de los ríos Anya y Mchique están ubicadas en los Andes centrales del Perú y son importantes, pues sus escurrimientos contribuyen a las descargas del río Mantaro, que permite el $35 \%$ de la generación del servicio eléctrico del país (Córdova, 2015). Estas cuencas son de pequeñas dimensiones, altas pendientes, con probabilidades de activación de procesos de remoción en masa, por lo cual se planteó conocer a profundidad los rasgos del relieve a través de un análisis cuantitativo con fines comparativos.

En este trabajo, el objetivo fue caracterizar y analizar la morfometría de las cuencas Anya y Mchique a través del cálculo de sus parámetros geomorfológicos para explicar sus respuestas hidrológicas.

\title{
Materiales y métodos
}

\author{
Área de estudio
}


Las cuencas de los ríos Anya y Mchique pertenecen a la zona central altoandina del Perú. Geográficamente, la cuenca del río Anya se sitúa en la margen izquierda del río Mantaro $\left(11.89^{\circ}-11.98^{\circ}\right.$ LS y $75.21^{\circ}-75.28^{\circ}$ LO); y la cuenca del río Mchique, en la margen derecha del río Mantaro $\left(11.86^{\circ}\right.$ a $11.97^{\circ}$ LS y $75.45^{\circ}$ a $75.38^{\circ}$ LO) (Figura 1 ).

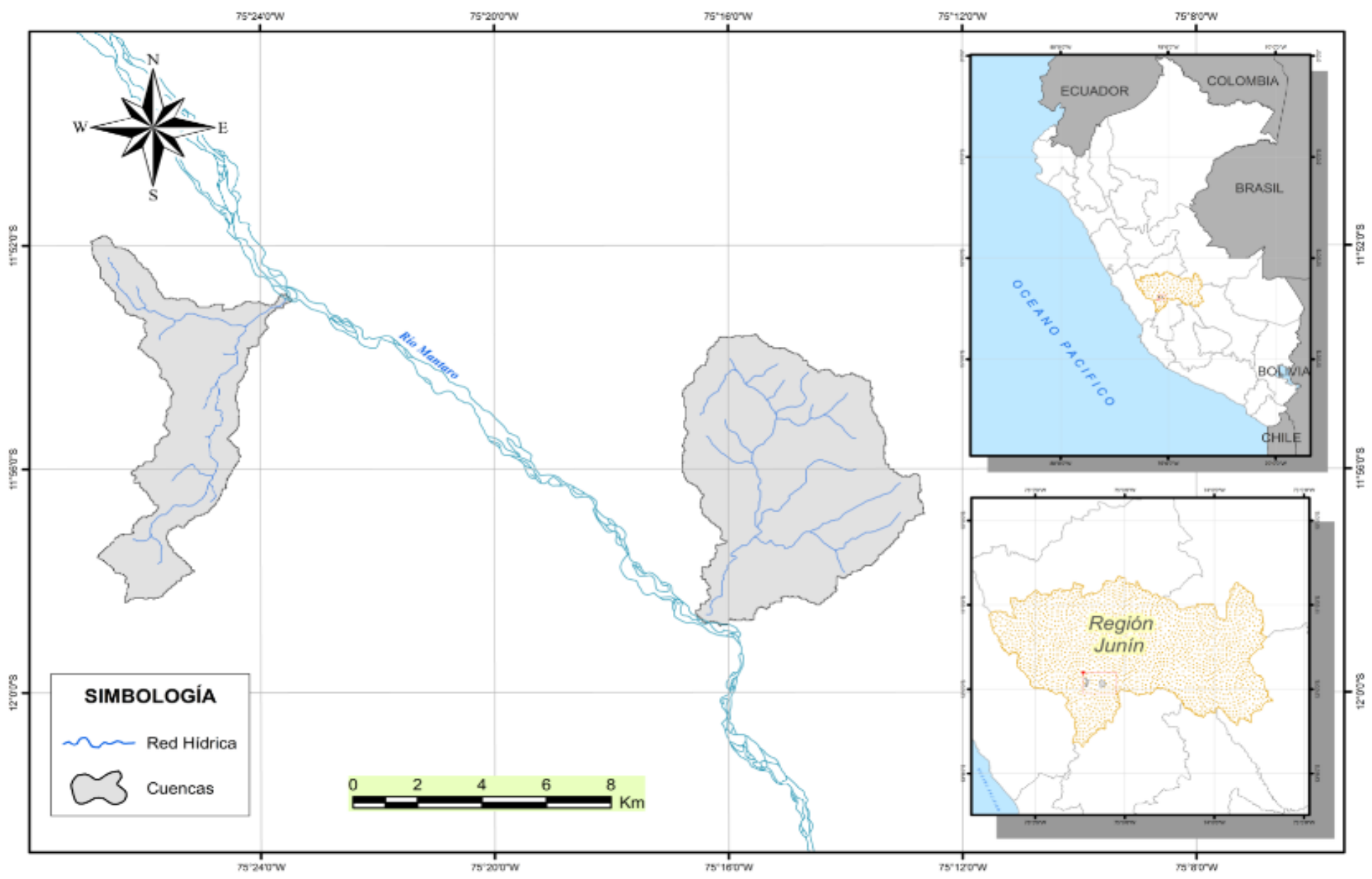

Figura 1. Mapa de ubicación de las cuencas Anya (derecha) y Mchique (izquierda). 


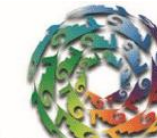

Tecnología y Ciencias $₫$ Agua

La zona de estudio comprende alturas entre 3000 y 4400 msnm; su clima es templado frío, con temperatura media anual de $10.5^{\circ} \mathrm{C}$. La precipitación acumulada multianual es 800 mm/año, con máximas precipitaciones en los meses de enero, febrero y marzo, disminuyendo fuertemente en abril hasta alcanzar sus valores mínimos en junio.

En las cuencas Anya y Mchique, las áreas de agricultura extensiva de secano destinada al autoconsumo son $40 \%$ y $51 \%$, respectivamente. Los cultivos representativos de la zona son papa, maíz, cebada, habas, olluco y avena. Además, el $29 \%$ y $40 \%$ le corresponde al herbazal rastrero andino de montañas y el $13.6 \%$ y $9 \%$ a los matorrales. Anya, a diferencia de Mchique, tiene plantaciones forestales de eucaliptos en un $12 \%$ del área de su cuenca.

\section{Fuente de datos y procesamiento}

Se seleccionó información cartográfica base (cartas topográficas del Instituto Geográfico Nacional (IGN) y mapa de ríos de la Autoridad Nacional de Agua (ANA)). Se accedió de forma remota a la plataforma https://vertex.daac.asf.alaska.edu/ para descargar el Modelo de Elevación Digital de terreno (MED) ALOS PALSAR, con resolución espacial de 12.5 m y código de adquisición AP_23821_FBD_F6940_RT1. Se recortó el MED ajustándolo al área de estudio y se hizo el proceso automático de delimitación de las cuencas con la opción ArcToolbox/Spatial Analyst 
Tools/Hydrology del programa ArcMap 10.5 (Figura 2). Después se procedió al cálculo de los parámetros básicos (área, perímetro, ancho, elevaciones, orden y longitud de las corrientes) de cada cuenca por separado. Finalmente, con base en los parámetros calculados con el programa Arc Map 10.5 en el paso previo y utilizando las ecuaciones de la Tabla 1, se calcularon elongación, alargamiento, factor de forma, coeficiente de compacidad, relación de bifurcación y densidad de drenaje.
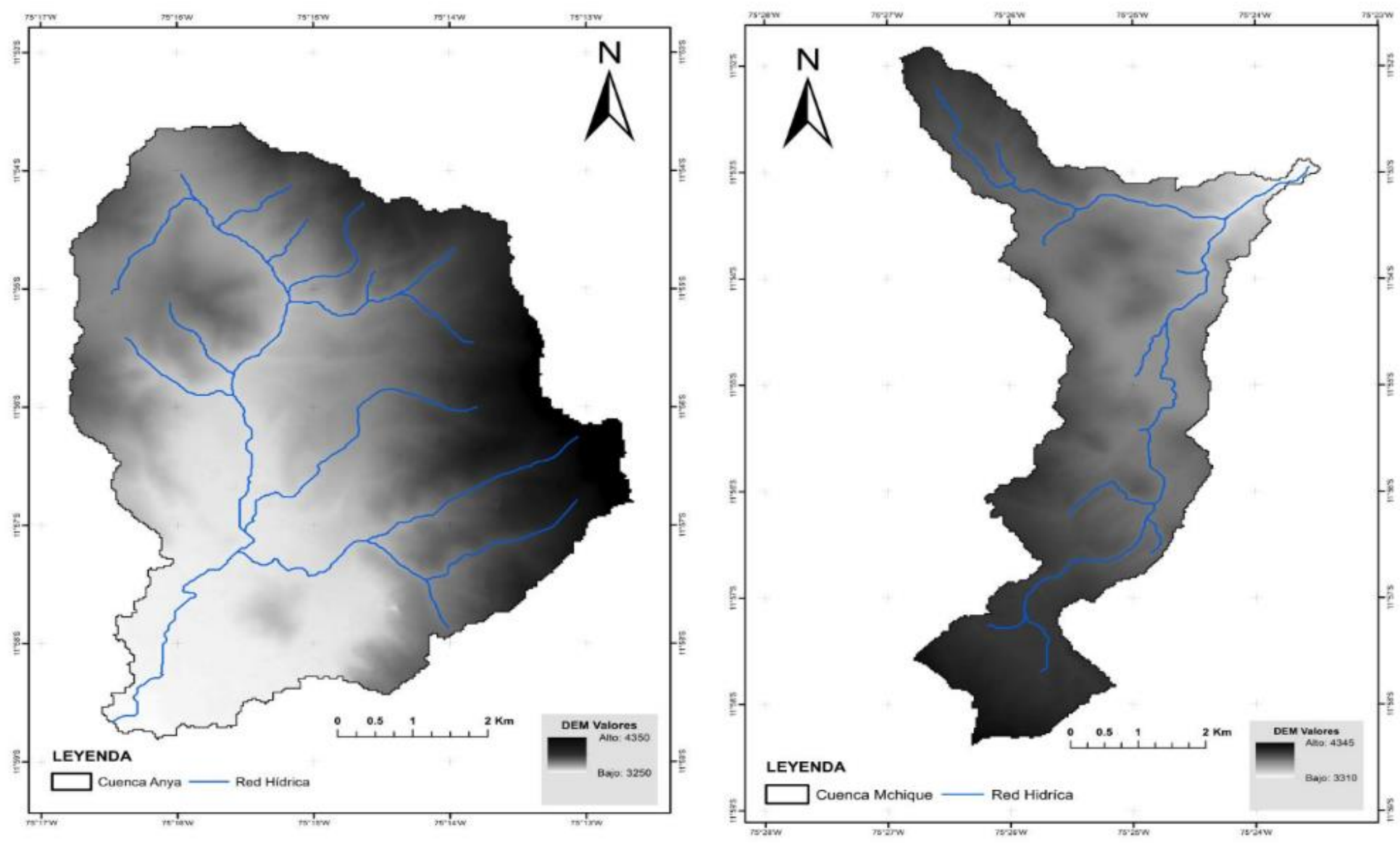

Figura 2. Modelos de elevación digital (MED) de las cuencas Anya y Mchique. 
Tabla 1. Ecuaciones para el cálculo de parámetros morfométricos en cuencas

\begin{tabular}{|c|c|c|c|}
\hline Parámetro & Símbolo & Fórmula/definición & Unidad \\
\hline \multicolumn{4}{|l|}{ Geometría de la cuenca } \\
\hline Área & A & Medido en el mapa SIG & $\mathrm{km}^{2}$ \\
\hline $\begin{array}{l}\text { Área del rectángulo equivalente } \\
\text { a la cuenca }\end{array}$ & $A_{r}$ & $\mathrm{~A}_{\mathrm{r}}=\mathrm{La}_{\text {máx }}$ & $\mathrm{km}^{2}$ \\
\hline $\begin{array}{l}\text { Lado mayor rectángulo } \\
\text { equivalente }\end{array}$ & Lmay & Lmay $=\sqrt{A}\left(\frac{K_{c}}{1.12}+\sqrt{\left(\frac{K_{c}}{1.12}\right)^{2}-1}\right.$ & $\mathrm{km}$ \\
\hline $\begin{array}{ll}\text { Lado menor rectángulo } \\
\text { equivalente }\end{array}$ & Lmen & Lmen $=\sqrt{A}\left(\frac{K_{c}}{1.12}-\sqrt{\left(\frac{K_{c}}{1.12}\right)^{2}-1}\right.$ & $\mathrm{km}$ \\
\hline Perímetro & $P$ & Medido en el mapa SIG & $\mathrm{km}$ \\
\hline Longitud & $L$ & Medido en el mapa SIG & $\mathrm{km}$ \\
\hline Ancho promedio & $a$ & $\mathrm{a}=\mathrm{A} / \mathrm{L}$ & $\mathrm{km}$ \\
\hline Ancho máximo & $a_{\text {máx }}$ & $\begin{array}{c}\text { Medido en el mapa SIG, } \\
\text { perpendicular a la longitud } \\
\text { de la cuenca }\end{array}$ & $\mathrm{km}$ \\
\hline Diámetro & $D$ & $D=\sqrt{\frac{4 A}{\pi}}$ & $\mathrm{km}$ \\
\hline $\begin{array}{l}\text { Perímetro de un círculo igual al } \\
\text { área de la cuenca }\end{array}$ & $P_{c}$ & $P c=\pi \sqrt{\frac{4 A}{\pi}}$ & $\mathrm{km}$ \\
\hline \multicolumn{4}{|c|}{ Gradiente y forma del relieve de la cuenca } \\
\hline Altitud mínima & $h$ & $\begin{array}{l}\text { Lectura de curvas de nivel } \\
\text { de la carta topográfica }\end{array}$ & msnm \\
\hline
\end{tabular}




\begin{tabular}{|c|c|c|c|}
\hline Altitud máxima & $H$ & $\begin{array}{l}\text { Lectura de curvas de nivel } \\
\text { de la carta topográfica }\end{array}$ & msnm \\
\hline Altitud media & $H_{m}$ & Estimada del SIG & msnm \\
\hline Relieve máximo & $R_{m}$ & $R_{m}=H-h$ & $\mathrm{msnm}$ \\
\hline Radio del relieve & $R$ & $R=R_{m} / L$ & $\begin{array}{c}\mathrm{msnm} / \mathrm{k} \\
\mathrm{m}\end{array}$ \\
\hline Relieve relativo & $R_{r}$ & $R_{r}=R_{m} / P$ & - \\
\hline \begin{tabular}{|l} 
Pendiente media (criterio de \\
Alvord)
\end{tabular} & $P_{m}$ & $\begin{array}{c}P_{m}=D \times L_{\text {total }} \text { curvas de } \\
\text { nivel } / \mathrm{A}\end{array}$ & $\%$ \\
\hline $\begin{array}{l}\text { Altitud de la naciente del cauce } \\
\text { principal }\end{array}$ & $H_{\text {nac }}$ & $\begin{array}{l}\text { Lectura de curvas de nivel } \\
\text { del mapa }\end{array}$ & msnm \\
\hline $\begin{array}{l}\text { Altitud del desagüe del cauce } \\
\text { principal }\end{array}$ & $h_{\text {des }}$ & $\begin{array}{l}\text { Lectura de curvas de nivel } \\
\text { del mapa }\end{array}$ & msnm \\
\hline $\begin{array}{l}\text { Altitud del cauce principal en su } \\
\text { origen como cauce de orden } \\
\text { mayor }\end{array}$ & $H_{\text {com }}$ & $\begin{array}{l}\text { Lectura de curvas de nivel } \\
\text { del mapa }\end{array}$ & msnm \\
\hline $\begin{array}{l}\text { Pendiente media del perfil } \\
\text { longitudinal del cauce principal } \\
\text { desde la naciente hasta la } \\
\text { desembocadura }\end{array}$ & $P_{\mathrm{cpm}}$ & $\begin{array}{l}\text { Lectura de curvas de nivel } \\
\text { del mapa }\end{array}$ & $\mathrm{m} / \mathrm{m}$ \\
\hline $\begin{array}{|lll|}\text { Relieve máximo del cauce } \\
\text { principal }\end{array}$ & $R_{\mathrm{mcp}}$ & $R_{\mathrm{mcp}}=H_{\mathrm{nac}}-h_{\mathrm{des}}$ & msnm \\
\hline Factor topográfico & $F_{t}$ & $F t=\mathrm{Rmcp} \sqrt{\mathrm{Pcpm}}$ & - \\
\hline Integral hipsométrica & $I H$ & $I H=(H m-h) /(H-h)$ & - \\
\hline Coeficiente de masividad & $C_{m}$ & $C_{m}=H_{m} / A$ & $\mathrm{~m} / \mathrm{km}^{2}$ \\
\hline Coeficiente orográfico & $C_{0}$ & $C_{o}=\left(H_{m} \text { en } \mathrm{km}\right)^{2} / A$ & - \\
\hline Número de rugosidad & $N R$ & $N R=R m A^{-0.5}$ & - \\
\hline
\end{tabular}


Tecnología y

Ciencias $₫$ Agua
2021, Instituto Mexicano de Tecnología del Agua

Open Access bajo la licencia CC BY-NC-SA 4.0

(https://creativecommons.org/licenses/by-nc-sa/4.0/)

\begin{tabular}{|c|c|c|c|}
\hline \multicolumn{4}{|c|}{ Forma de la cuenca } \\
\hline Orientación de la cuenca & - & $\begin{array}{l}\text { Dirección con respecto al } \\
\text { norte geográfico. Se mide } \\
\text { sobre el mapa }\end{array}$ & $\circ$ \\
\hline Elongación & $E$ & $E=1.129 \frac{A^{0.5}}{L}$ & - \\
\hline Coeficiente de compacidad & $K_{c}$ & $K_{c}=0.28 \frac{P}{\sqrt{A}}$ & - \\
\hline Índice de alargamiento & $I_{a}$ & $I a=\frac{L}{a_{\text {máx }}}$ & - \\
\hline Índice de homogeneidad & $I_{h}$ & $I_{h}=A / A_{r}$ & - \\
\hline Factor de forma & $F_{f}$ & $F f=\frac{A}{L c p^{2}}$ & - \\
\hline Alargamiento de Caquot & $A_{c}$ & $A c=\frac{L}{A^{0.5}}$ & - \\
\hline Radio de elongación & $R e$ & $R_{e}=D / L$ & - \\
\hline Radio de circularidad & $R C$ & $R C=\frac{4 \pi A}{P^{2}}$ & - \\
\hline Índice de forma & IF & $I F=P / 2 \pi A$ & - \\
\hline $\begin{array}{l}\text { Índice entre el largo y el área de } \\
\text { la cuenca }\end{array}$ & ICo & $I C o=D / A$ & - \\
\hline Coeficiente de forma & $C_{f}$ & $\begin{array}{l}C_{f}=a m / L a m=\text { ancho } \\
\text { medio de la cuenca }=a\end{array}$ & - \\
\hline Coeficiente de redondez & $C_{r}$ & $C r=\frac{\pi L^{2}}{4 A}$ & - \\
\hline \multicolumn{4}{|l|}{ Extensión de la red de drenaje } \\
\hline Longitud del cauce principal & $L_{c p}$ & Medido en el mapa SIG & $\mathrm{km}$ \\
\hline Orden mayor del río principal & - & Del mapa SIG & - \\
\hline
\end{tabular}




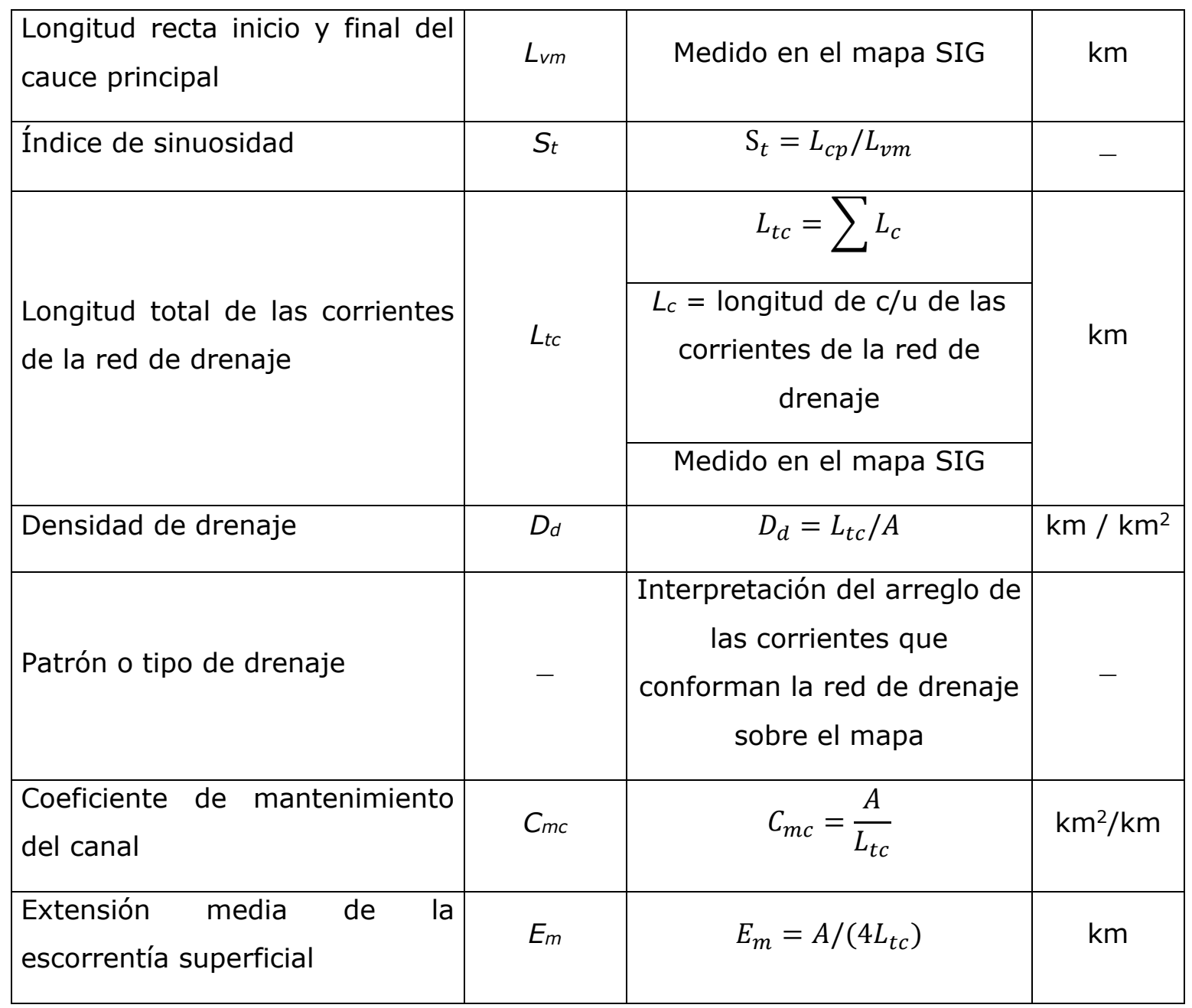

Se representó la curva hipsométrica de las cuencas, que es una curva de doble eje donde la ordenada representa la cota altitudinal en msnm (con una equidistancia de $200 \mathrm{~m}$ ) y la abscisa es el área por encima o por debajo de una cota (\%), tal como lo estableció Strahler (1964). Esta curva es importante porque nos proporciona información sobre las etapas del paisaje y los procesos de erosión que ocurren en la cuenca (Cruz et al., 2015). El tiempo de concentración $\left(T_{c}\right)$ se calculó como el valor promedio obtenido de fórmulas empíricas desarrolladas para diferentes condiciones. 


\section{Resultados y discusión}

Las superficies de las cuencas Anya y Mchique son $48.03 \mathrm{~km}^{2}$ y 28.55 $\mathrm{km}^{2}$; sus perímetros son de $31.59 \mathrm{~km}$ y $39.33 \mathrm{~km}$, respectivamente, siendo la cuenca Mchique de mayor perímetro. La orientación de Anya es hacia el suroeste y Mchique está orientada hacia el noreste. El rectángulo equivalente de la cuenca Anya tiene por lados $4.11 \mathrm{~km}$ y $11.68 \mathrm{~km}$, y los de Mchique $1.58 \mathrm{~km}$ y $18.08 \mathrm{~km}$, lo que nos da una idea de la extensión longitudinal de Mchique. Las alturas medias son 3700 msnm y 3831.42 msnm en Anya y Mchique, siendo las pendientes medias $\left(P_{m}\right)$ de las cuencas iguales a $20.9 \%$ y $15.8 \%$, respectivamente, que corresponden a relieves fuertemente accidentados $\left(P_{m}>15 \%\right)$ típicos de cordillera, las que influyen en la velocidad de la escorrentía superficial (Pérez, 1979). Por un lado, respecto a la curva hipsométrica y su forma, se infiere que la cuenca Anya se encuentra en una etapa de madurez y equilibrio, representando una actividad erosiva media (curva tipo B); por otro lado, la cuenca Mchique es joven, reflejando un gran poder erosivo y un potencial de alta actividad (curva tipo A). Las altitudes medianas son 3 600 msnm y 3800 msnm para Anya y Mchique, respectivamente.

En cuanto al relieve, los valores máximos de cada una de las cuencas Anya y Mchique son 1080 m y 932 m, y del cauce principal de 
sus ríos son $273.23 \mathrm{~m}$ y $728.52 \mathrm{~m}$, respectivamente; los coeficientes de masividad $(\mathrm{Cm})$ son 77.04 y $134.20 \mathrm{~m} / \mathrm{km}^{2}$, respectivamente; los coeficientes orográficos son 0.29 y 0.51 , respectivamente; y los números de rugosidad de Melton (NR) resultaron 155.84 y 174.43, respectivamente. Los radios de relieve $(R)$, definido como el cociente del relieve máximo de la cuenca entre la longitud de la cuenca, resultaron de $120 \mathrm{msnm} / \mathrm{km}$ en Anya y de 83.44 msnm/km en Mchique (Tabla 2). El relieve influye de forma significativa en la velocidad de los procesos erosivos en una cuenca, ya que determina la dirección e intensidad del movimiento de agua y sedimentos (Schumm, 1977). Los resultados reflejan que la cuenca del río Mchique tiene los valores más altos del coeficiente de masividad $(\mathrm{Cm})$, coeficiente orográfico y número de rugosidad de Melton (NR), respectivamente; sin embargo, en los dos casos, los valores obtenidos son típicos de cordillera y representan zonas montañosas, con relieves muy accidentados y de un alto potencial erosivo. El radio de relieve es mayor en la cuenca Anya por su mayor diferencia topográfica y menor longitud de cuenca que Mchique.

Tabla 2. Resultados de cálculo de parámetros morfométricos en las cuencas Anya y Mchique.

\begin{tabular}{|l|c|r|r|}
\hline \multirow{2}{*}{ Parámetro } & \multirow{2}{*}{ Unidad } & \multicolumn{2}{c|}{ Magnitud } \\
\cline { 3 - 4 } & & Anya & Mchique \\
\hline \multicolumn{2}{|c|}{ Geometría de la cuenca } \\
\hline Área & $\mathrm{km}^{2}$ & 48.03 & 28.55 \\
\hline $\begin{array}{l}\text { Área del rectángulo equivalente a la } \\
\text { cuenca }\end{array}$ & $\mathrm{km}^{2}$ & 48.00 & 28.57 \\
\hline
\end{tabular}




\begin{tabular}{|c|c|c|c|}
\hline Lado mayor rectángulo equivalente & $\mathrm{km}$ & 11.68 & 18.08 \\
\hline Lado menor rectángulo equivalente & $\mathrm{km}$ & 4.11 & 1.58 \\
\hline Perímetro & $\mathrm{km}$ & 31.59 & 39.33 \\
\hline Longitud & $\mathrm{km}$ & 9.00 & 11.17 \\
\hline Ancho promedio & $\mathrm{km}$ & 5.34 & 2.56 \\
\hline Ancho máximo & $\mathrm{km}$ & 7.77 & 2.84 \\
\hline Diámetro & $\mathrm{km}$ & 7.82 & 6.03 \\
\hline $\begin{array}{l}\text { Perímetro de un círculo igual al área } \\
\text { de la cuenca }\end{array}$ & $\mathrm{km}$ & 24.57 & 18.94 \\
\hline \multicolumn{4}{|c|}{ Gradiente y forma del relieve de la cuenca } \\
\hline Altitud mínima & msnm & 3240 & 3308 \\
\hline Altitud máxima & msnm & 4320 & 4240 \\
\hline Altitud media & msnm & 3700 & 3831.4 \\
\hline Relieve máximo & msnm & 1080 & 932 \\
\hline Radio del relieve & $\mathrm{msnm} / \mathrm{km}$ & 120 & 83.44 \\
\hline Relieve relativo & - & 3.8 & 2.1 \\
\hline Pendiente media (Alvord) & $\%$ & 20.9 & 15.8 \\
\hline $\begin{array}{l}\text { Altitud de la naciente del cauce } \\
\text { principal }\end{array}$ & msnm & 3529.2 & 4064 \\
\hline $\begin{array}{l}\text { Altitud del desagüe del cauce } \\
\text { principal }\end{array}$ & msnm & 3256 & 3335.5 \\
\hline $\begin{array}{l}\text { Altitud del cauce principal en su } \\
\text { origen como cauce de orden mayor }\end{array}$ & msnm & 3298 & 3424 \\
\hline $\begin{array}{l}\text { Pendiente media del perfil } \\
\text { longitudinal del cauce principal desde } \\
\text { la naciente hasta la desembocadura }\end{array}$ & $\mathrm{m} / \mathrm{m}$ & 0.03 & 0.06 \\
\hline
\end{tabular}




\begin{tabular}{|c|c|c|c|}
\hline Relieve máximo del cauce principal & msnm & 273.23 & 728.52 \\
\hline Factor topográfico & - & 43.2 & 178.45 \\
\hline Integral hipsométrica & - & 0.43 & 0.56 \\
\hline Coeficiente de masividad & $\mathrm{m} / \mathrm{km}^{2}$ & 77.04 & 134.2 \\
\hline Coeficiente orográfico & - & 0.29 & 0.51 \\
\hline Número de rugosidad & - & 155.84 & 174.43 \\
\hline \multicolumn{4}{|c|}{ Forma de la cuenca } \\
\hline Orientación de la cuenca & $\circ$ & $19^{\circ} \mathrm{SO}$ & $28^{\circ} \mathrm{NE}$ \\
\hline Elongación & - & 0.87 & 0.54 \\
\hline Coeficiente de compacidad & - & 1.28 & 2.06 \\
\hline Índice de alargamiento & - & 1.16 & 3.93 \\
\hline Índice de homogeneidad & - & 1.001 & 0.999 \\
\hline Factor de forma & - & 0.38 & 0.2 \\
\hline Alargamiento de Caquot & - & 1.3 & 2.09 \\
\hline Radio de elongación & - & 0.87 & 0.54 \\
\hline Radio de circularidad & - & 0.6 & 0.23 \\
\hline Índice de forma & - & 0.1 & 0.22 \\
\hline $\begin{array}{l}\text { Índice entre el largo y el área de la } \\
\text { cuenca }\end{array}$ & - & 0.16 & 0.21 \\
\hline Coeficiente de forma & - & 0.59 & 0.23 \\
\hline Coeficiente de redondez & - & 1.32 & 3.43 \\
\hline \multicolumn{4}{|c|}{ Extensión de la red de drenaje } \\
\hline Longitud del cauce principal & $\mathrm{km}$ & 11.3 & 12 \\
\hline Orden mayor del río principal & - & 3 & 3 \\
\hline
\end{tabular}


Tecnología y

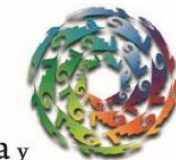

Ciencias ฐึgua
2021, Instituto Mexicano de Tecnología del Agua

Open Access bajo la licencia CC BY-NC-SA 4.0

(https://creativecommons.org/licenses/by-nc-sa/4.0/)

\begin{tabular}{|l|r|r|r|}
\hline $\begin{array}{l}\text { Longitud recta inicio y final del cauce } \\
\text { principal }\end{array}$ & $\mathrm{km}$ & 9.3 & 10.3 \\
\hline Índice de sinuosidad & - & 1.22 & 1.17 \\
\hline $\begin{array}{l}\text { Longitud total de las corrientes de la } \\
\text { red de drenaje }\end{array}$ & $\mathrm{km}$ & 41.12 & 25.56 \\
\hline Densidad de drenaje & $\mathrm{km} / \mathrm{km}^{2}$ & 0.86 & 0.9 \\
\hline Patrón o tipo de drenaje & $\mathrm{km}{ }^{2} / \mathrm{km}$ & 1.17 & 1.12 \\
\hline $\begin{array}{l}\text { Coeficiente de mantenimiento del } \\
\text { canal }\end{array}$ & $\mathrm{km}$ & 0.29 & Sub-dendrítico \\
\hline $\begin{array}{l}\text { Extensión media de la escorrentía } \\
\text { superficial }\end{array}$ & & & \\
\hline
\end{tabular}

Con respecto a los perfiles longitudinales de los ríos Anya y Mchique, se obtuvieron pendientes promedio de 3 y $6 \%$, respectivamente; y longitudes de corrientes principales iguales a 11.3 y $12 \mathrm{~km}$, respectivamente; el río Anya tiene un lecho conformado por tres zonas: una zona alta (de mayores pendientes en el sector cordillerano), media y baja (coincidente con el valle). El río Mchique se desarrolla a partir de un macizo rocoso, con una zona vadosa (bofedal) en la mitad de su recorrido, y su pendiente muestra cambios abruptos debido al control litológico y estructural, inclusive muy cerca de su confluencia con el río Mantaro. Con respecto al lecho de los ríos, Anya y Mchique muestran algunas diferencias: el río Anya tiene un lecho poco rocoso y parcialmente vegetado, siendo sinuoso en la parte baja; el río Mchique no es sinuoso y posee un lecho rocoso en toda su trayectoria (Grados, 2012), por lo que presenta acarreo de materiales de mayor peligrosidad. 
Los coeficientes de compacidad $K_{c}$ de las cuencas Anya y Mchique son 1.28 y 2.06, respectivamente; esto significa que la forma de la cuenca Anya es más parecida a un circulo $\left(K_{c}=1\right)$ y la cuenca Mchique es más alargada. Respecto al factor de forma $\left(F_{f}\right)$, que es el cociente entre el área de la cuenca y el cuadrado de la longitud de la cuenca, resultan 0.38 y 0.20 , respectivamente, lo que sugiere formas distintas a la de un círculo de $F_{f}=0.7854$, y que las cuencas Anya y Mchique son oblongas, siendo la cuenca Mchique mucho más alargada que la cuenca Anya. La forma alargada es menos susceptible a las inundaciones en situaciones de precipitación extrema, pero la posibilidad de que la lluvia cubra toda la extensión, incluidos los ríos tributarios, también es baja, pues la inundación golpea el río principal en varios puntos (Medeiros et al., 2019).

El radio de circularidad $\left(R_{c}\right)$ está influenciado por la longitud y frecuencia de las corrientes, estructura geológica, cobertura del suelo, clima y pendiente de la cuenca (Waikar \& Nilawar, 2014). Los valores altos del radio de circularidad indican una etapa de madurez de la topografía (Rai et al., 2017). En este caso, los radios de circularidad son 0.60 y 0.23 para Anya y Mchique, los cuales son valores bajos y son indicativos de que la topografía está en formación (juventud) en ambas cuencas, siendo Mchique más joven que Anya.

El radio de elongación $\left(R_{e}\right)$ es el diámetro de un círculo de área igual al área de drenaje de la cuenca dividido entre la máxima longitud de la cuenca. Tiene valores que varían entre 0.6 y 1.0 , en un rango amplio de climas y geología. Cuando $R_{e}<0.88$ se considera elongado y usualmente está asociado con relieves y pendientes pronunciados (Strahler, 1964; citado por Kabite $\&$ Gessese, 2018). Se obtuvo $R_{e}$ de 0.87 y 0.54 , lo que 
Tecnología y

Ciencias $₫$ Agua
2021, Instituto Mexicano de Tecnología del Agua

Open Access bajo la licencia CC BY-NC-SA 4.0

(https://creativecommons.org/licenses/by-nc-sa/4.0/)

significa que la cuenca Anya es oval oblonga y la cuenca Mchique tiene mayor elongación.

La red de drenaje contiene cauces o corrientes de agua de recorrido corto y régimen intermitente en grandes porcentajes (51.9 \% Anya y 47.4 $\%$ Mchique) con longitud total de las corrientes de $41.12 \mathrm{~km}$ y $25.56 \mathrm{~km}$ en Anya y Mchique, respectivamente. Horton (1945) introdujo la densidad de drenaje $\left(D_{d}\right)$ como un importante indicador de escala lineal de los elementos de forma de la tierra y la erosión de la topografía de las corrientes, la cual se obtiene del cociente de la longitud del total de corrientes $(\mathrm{km})$ entre el área de la cuenca $\left(\mathrm{km}^{2}\right)$. La densidad de drenaje es un indicador de la eficiencia de drenaje de las cuencas y varía de 0.5 $\mathrm{km} / \mathrm{km}^{2}$ en cuencas con drenaje pobre, y $3.5 \mathrm{~km} / \mathrm{km}^{2}$ a más para cuencas bien drenadas (Mejía, 2012). Las $D_{d}$ de las cuencas Anya y Mchique son 0.86 y $0.95 \mathrm{~km} / \mathrm{km}^{2}$, respectivamente, correspondiéndoles una baja densidad debido a la corta longitud de sus cauces y poca extensión de sus cuencas de drenaje. En cuencas de mayor tamaño, la baja densidad de drenaje conduce a inferir una textura de drenaje gruesa, mientras que la alta densidad de drenaje conduce a una textura de drenaje fina, un alto escurrimiento y un potencial de erosión del área de la cuenca (Kabite \& Gessesse, 2018). Ambas cuencas tienen un sistema hidrogeomorfológico de orden 3, con patrones de drenaje dendrítico en Anya y subdendrítico en Mchique; el patrón de drenaje hidrológicamente dendrítico refleja homogeneidad en textura de los estratos de subsuelo y el patrón subdendrítico, control estructural (Banerjee, Singh, \& Pratap, 2017).

Las pequeñas áreas de estas cuencas implican tiempos de concentración cortos, en las cuales toda el área contribuye a la escorrentía superficial; se espera que los hidrogramas sean de picos pronunciados y 
corta duración, es decir, en presencia de una tormenta de extensión considerable, toda la cuenca se activa y aporta agua, alcanzando el gasto de equilibrio. Los resultados de los tiempos de concentración $\left(T_{c}\right)$ de las cuencas Anya y Mchique son 3.20 y 2.21 horas, respectivamente, obtenidas del promedio de las ecuaciones consideradas; el valor mayor en Anya se debe a su menor pendiente, $3 \%$, frente al $6 \%$ de Mchique, no obstante tener una menor longitud de su corriente principal de 11.3 $\mathrm{km}$ con respecto a Mchique, que es de $12.0 \mathrm{~km}$ (Tabla 3).

Tabla 3. Tiempos de concentración en horas de las cuencas Anya y Mchique.

\begin{tabular}{|l|c|c|c|}
\hline \multicolumn{1}{|c|}{$\begin{array}{c}\text { Tiempos de } \\
\text { concentración (hr) }\end{array}$} & Ecuación & $\begin{array}{c}\text { Cuenca } \\
\text { Anya }\end{array}$ & $\begin{array}{c}\text { Mchique } \\
\text { Mchiqu }\end{array}$ \\
\hline Giandiotti & $T_{c}=\frac{4 \sqrt{A}+1.5 L}{25.3 \sqrt{S L}}$ & 3.49 & 1.82 \\
\hline Kirpich & $T_{c}=0.06628\left[\frac{L^{0.77}}{S^{0.385}}\right]$ & 1.84 & 1.32 \\
\hline California (U.S.B.R.) & $T_{c}=\left[\frac{0.87075 L^{3}}{H}\right]^{0.385}$ & 1.84 & 1.32 \\
\hline Bransby - Williams & $T_{c}=14.6 L A^{-0.1} S^{-0.2} / 60$ & 3.98 & 3.66 \\
\hline Passini & $T_{c}=\frac{\left[0.108(A L)^{1 / 3}\right]}{S^{0.5}}$ & 5.86 & 3.07 \\
\hline Pérez & $T_{c}=\frac{L}{72\left(\frac{H}{L}\right)^{0.6}}$ & 1.52 & 0.90 \\
\hline
\end{tabular}




\begin{tabular}{|l|c|c|c|}
\hline Témez & $T_{c}=0.3\left[\frac{L}{S^{0.25}}\right]^{0.76}$ & 3.89 & 3.38 \\
\hline Desviación estándar & & 0.95 & 0.29 \\
\hline Varianza & & 0.91 & 0.09 \\
\hline Máximo & & 5.86 & 3.66 \\
\hline Mínimo & & 1.52 & 0.90 \\
\hline Promedio final & & $\mathbf{3 . 2 0}$ & $\mathbf{2 . 2 1}$ \\
\hline
\end{tabular}

\section{Conclusiones}

El análisis morfométrico de cuencas es la base de los estudios hidrológicos como parte de los estudios en recursos hídricos. El uso de los sistemas de información geográfica, específicamente el Arc GIS y técnicas computacionales, permiten la obtención de resultados para un entendimiento rápido de la dinámica física de la red de drenaje de cuencas hidrográficas, en este caso de los ríos Anya y Mchique, afluentes del río Mantaro en Junín, Perú.

Las cuencas estudiadas poseen paisajes montañosos con fuertes desniveles, en las que, a través del análisis morfométrico, se concluye que sus capacidades erosivas se ven acentuadas por sus pendientes, más que por sus índices de compacidad y elongación; y sus capacidades de acarreo y transporte de materiales por la composición estructural del 
lecho de sus ríos. Por los parámetros identificados, se infiere que en ambas cuencas existe un riesgo de avenidas, moderadas en Anya y bajas en Mchique, existiendo similitud hidrológica entre ambas cuencas.

La información reportada en este estudio puede servir a otros de índole hidrológico e hidráulico; por ejemplo, en modelos de generación de escorrentía, además, utilizando la teoría de similitud hidráulica es posible aplicar los resultados obtenidos trasladando modelos de pequeña escala a prototipos de gran escala para hacer los comparativos necesarios.

\section{Agradecimientos}

Los autores expresamos nuestro agradecimiento a la Universidad San Cristóbal de Huamanga y al Programa de Doctorado en Recursos Hídricos de la Universidad Nacional Agraria La Molina por permitirnos desarrollar labor docente y de investigación.

\section{Referencias}

Banerjee, A., Singh, P., \& Pratap, K. (2017). Morphometric evaluation of Swarnrekha watershed, Madhya Pradesh, India: An integrated GISbased approach. Journal Applied Water Science, 7, 1807. Recuperado de https://doi.org/10.1007/s13201-015-0354-3.

Córdova, M. (2015). Estimación de caudales medios naturalizados en la cuenca del río Mantaro mediante el método de regionalización estadística (tesis para optar el título de Ingeniero Civil), Pontificia Universidad Católica del Perú. Lima, Perú. Recuperado de http://tesis.pucp.edu.pe/repositorio/handle/20.500.12404/6273 
Cruz, B., Gaspari, F. J., Rodriguez, A. M., Carrillo, F. M., \& Telles, J. (2015). Análisis morfométrico de la cuenca hidrográfica del río Cuale, Jalisco, México. Revista Investigación y Ciencia de la Universidad Autónoma de Aguascalientes, (64), 26-34. Recuperado de http://www.redalyc.org/pdf/674/67441039004.pdf

García, E., \& Otto, M. (2015). Caracterización ecohidrológica de humedales alto andinos usando imágenes de satélite multitemporales en la cabecera de cuenca del río Santa, Ancash, Perú. Ecología Aplicada, 14, 115-125.

Grados, D. (2012). Estudio de avenidas en las cuencas del Anya y Mchique, departamento de Junín, Perú (Período 2011-2012) (tesis para optar el título de Ingeniero Agrícola), Universidad Nacional Agraria La Molina, Lima, Perú.

Horton, R. E. (1945). Erosional development streams and their drainage basins: Hidrophysical approach to quantitative morphology. Geological Society of America Bulletin, 56, 275-280.

Jardí, M. (1985). Forma de una cuenca de drenaje. Análisis de las variables morfométricas que nos la definen. Revista de Geografía, (19), 41-68.

Kabite, G., \& Gessesse, B. (2018). Hydro-geomorphological characterization of Dhidhessa River Basin, Etiopia. International Soil and Water Conservation Research Journal, 6(2), 175-183. Recuperado de https://doi.org/10.1016/j.iswcr.2018.02.003

Liniger, H., Weingartner, R., \& Grosjean, M. (1998). Mountains of the World: Water towers for the 21st Century. Berne, Switzerlad: Centre for Development and Environment. 
Medeiros, F., Lima, J., Silva, R., Pereira, S., Freitas, A., Lima, A., Lima, L., Silva, J. L., \& Monte, A. (2019). Analysis of morphometric variables of river Espinharas hydrographic sub-basin using geographic information system. Journal of Experimental Agriculture International, 31(6), 1-18. Recuperado de https://doi.org/10.9734/jeai/2019/v31i63008

Mejía, A. (2012). Hidrología aplicada. Lima, Perú: Universidad Nacional Agraria La Molina.

Miller, V. C. (1953). A quantitative geomorphic study of drainage in the Clinch Mountain area, Virginia and Tennessee. Office of Naval Research, Geography Branch, Project NR 389-042, Technical Report, 3. Columbia, USA: Columbia University.

Oruonye, E. D., Ezekiel, B. B, Atiku, H. G., Baba, E., \& Musa, N. I. (2015). Drainage basin morphometric parameters of river Lamurde: Implication for hydrologic and geomorphic processes. Journal of Agriculture and Ecology Research International, 5(2), 1-11. DOI: 10.9734/JAERI/2016/22149

Pérez, J. (1979). Fundamentos del ciclo hidrológico. Caracas, Venezuela: Universidad Central de Venezuela, Facultad de Ingeniería, Departamento de Meteorología e Hidrología.

Rai, P. K., Mohan, K., Mishra, S., Ahmad, A., \& Mishra, V. A. (2017). A GIS-based approach in drainage morphometric analysis of Kanhar River Basin, India. Journal Applied Water Science, 7, 217. Recuperado de https://doi.org/10.1007/s13201-014-0238-y

Schumm, A. (1977). The fluvial system. New York, USA: John Wiley \& Sons. 
Strahler, A. N. (1964). Quantitative geomorphology of drainage basins and channel networks. In: Chow, V. T. (ed.). Handbook of applied hydrology (pp. 439-476). New York, USA: McGraw- Hill.

Vieceli, N., Bortolin, T. A., Abritta-Mendes, L., Bacarim, G., Cemin, G., \& Schneider, V. E. (2015). Morphometric evaluation of watersheds in Caxias do Sul City, Brazil, using SRTM (DEM) data and GIS. Environmental Earth Sciences, 73(9), 5677-5685. Recuperado de https://doi.org/10.1007/s12665-014-3823-3

Waikar, M. L., \& Nilawar, A. P. (2014). Morphometric analysis of a drainage basin using Geographical Information System: A case study. International Journal of Multidisciplinary and Current Research, (2), 179-184. Recuperado de http://ijmcr.com/wpcontent/uploads/2014/02/Paper32179-184.pdf 